\title{
Conditional Pten knockout in parvalbumin- or somatostatin-positive neurons sufficiently leads to autism-related behavioral phenotypes
}

\author{
Sangyep Shin ${ }^{\dagger}$, Andrea Santi ${ }^{\dagger}$ and Shiyong Huang ${ }^{*}$ (B)
}

\begin{abstract}
Disrupted GABAergic neurons have been extensively described in brain tissues from individuals with autism spectrum disorder (ASD) and animal models for ASD. However, the contribution of these aberrant inhibitory neurons to autismrelated behavioral phenotypes is not well understood. We examined ASD-related behaviors in mice with conditional Pten knockout in parvalbumin (PV)-expressing or somatostatin (Sst)-expressing neurons, two common subtypes of GABAergic neurons. We found that mice with deletion of Pten in either PV-neurons or Sst-neurons displayed social deficits, repetitive behaviors and impaired motor coordination/learning. In addition, mice with one copy of Pten deletion in PV-neurons exhibited hyperlocomotion in novel open fields and home cages. We also examined anxiety behaviors and found that mice with Pten deletion in Sst-neurons displayed anxiety-like behaviors, while mice with Pten deletion in PV-neurons exhibited anxiolytic-like behaviors. These behavioral assessments demonstrate that Pten knockout in the subtype of inhibitory neurons sufficiently gives rise to ASD-core behaviors, providing evidence that both PV- and Sst-neurons may play a critical role in ASD symptoms.
\end{abstract}

Keywords: Autism, Social deficits, Repetitive behaviors, Mouse model, Pten, Motor deficits

\section{Introduction}

Inhibitory neurons are highly impacted in autism spectrum disorder (ASD) [1-3], a neurodevelopmental condition characterized by symptoms of difficulty in communication, deficits in social interaction, and the presence of restricted/repetitive behaviors [4]. Soma-targeting parvalbumin (PV)-expressing neurons (PV-neurons) and dendritic-targeting somatostatin (Sst)expressing neurons (Sst-neurons) are two prominent subtypes of inhibitory neurons in the cortex [5]. Studies of postmortem human brain tissues have demonstrated aberrant inhibitory neurons, mainly PV-neurons, in the brains of individuals with autism [6-9]. Disrupted

\footnotetext{
*Correspondence: shuang@hussmanautism.org

'Sangyep Shin and Andrea Santi have contributed equally

Program in Neuroscience, Hussman Institute for Autism, Baltimore, MD 21201, USA
}

inhibitory neurons have also been extensively reported in animal models for autism [10-12]. However, the contribution of each subtype of inhibitory neurons to autism symptoms is largely unknown. Thus, evaluating the role of PV-neurons and Sst-neurons in autism behaviors is crucial for the development of treatments.

The autism-risk gene, phosphatase and tensin homolog on chromosome ten (PTEN), originally identified as a tumor suppressor gene, negatively regulates cell proliferation and growth by downregulating the phosphatidylinositol 3-kinase/AKT/mammalian target of rapamycin (PI3K/AKT/mTOR) pathway [13-16]. PTEN germline mutations have been identified in individuals with ASD $[17,18]$, and may account for up to $12 \%$ of ASD cases [19-21]. Pten is widely expressed in both glutamatergic and GABAergic neurons during development and adulthood [22, 23]. Since deletion of Pten is embryonically lethal, mice with brain-region-specific Pten knockout or 
Pten haploinsufficiency have been investigated. These transgenic mice exhibited autism-related behaviors, including deficits in social interaction, repetitive behaviors, hyperlocomotion, and anxiety-like behaviors [2430]. However, whether conditional knockout of Pten in subtypes of inhibitory neurons causes autism-behavioral phenotypes remains elusive.

We used the Cre/loxp recombination system to generate mice with Pten knockout in PV-neurons or Sstneurons, and conducted a battery of behavioral tests to examine autism-related behavioral phenotypes in these mice. We found that conditional knockout Pten in PVneurons or Sst-neurons is sufficient to result in autismcore symptoms, including social deficits and repetitive behaviors. We also showed that Pten in PV-neurons and Sst-neurons is crucial for motor coordination and learning. Finally, deletion of Pten in PV-neurons induces hyperlocomotion and anxiolytic-like behaviors whereas deletion of Pten in Sst-neurons causes anxiety-like behaviors. Collectively, Pten mutation in PV-neurons and Sstneurons results in autism-related behavioral phenotypes.

\section{Results \\ Deletion of Pten in PV-neurons or Sst-neurons leads to social deficits}

First, we examined the effect of conditional Pten knockout in parvalbumin-expressing neurons (PV-neurons) on social behaviors. We specifically deleted Pten in PV-neurons by crossing PV-Cre mice with Pten ${ }^{\text {flox }}$ mice to generate PV-Cre ${ }^{+/+} /$Pten $^{\mathrm{f} /+}$ mice (See Methods for details). Using these mice as breeders, we obtained offspring littermates with three genotypes, PV-Cre ${ }^{+/+} /$Pten $^{+/+}$ (PV-Pten-WT), PV-Cre ${ }^{+/+} /$Pten $^{\mathrm{fl} /+}$ (PV-Pten-Het), and $\mathrm{PV}-\mathrm{Cre}^{+/+} / \mathrm{Pten}^{\mathrm{f} / \mathrm{fl}}$ (PV-Pten-KO). These three types of mice all have homozygous Cre but different copies of Pten in PV-neurons. Using immunohistochemistry and
Western blot, we confirmed that Pten was specifically reduced and deleted in PV-positive neurons of PV-PtenHet and PV-Pten-KO mice, respectively (Additional file 1: Figure S1). Sociability and social novelty preference in these mice were assessed using modified threechamber social tests (Fig. 1a). Firstly, we confirmed that there was no pre-existing side preference in the PVPten mice (Additional file 1: Figure S3A). As shown in Fig. 1b, single or two copies of Pten deletion in PV-neurons did not change the social approach behaviors. All three genotypes of PV-Pten mice spent more time with social partners than with objects in the sociability test. The preference index was not different among the three groups of PV-Pten mice (Fig. 1b). In contrast, knockdown or knockout of Pten in PV-neurons impaired the social novelty preference. In the social novelty test, only PVPten-WT mice spent more time interacting with novel social partners (Fig. 1c). The preference index for social novelty decreased in PV-Pten-Het and PV-Pten-KO mice compared to that in PV-Pten-WT mice (Fig. 1c).

Next, we evaluated the effect of conditional Pten knockout in somatostatin-expressing neurons (Sst-neurons) on social behaviors. Using the same breeding strategy for PV-Pten mice, we obtained Sst-Pten littermates with three genotypes, Sst-Cre ${ }^{+/+} /$Pten $^{+/+}$(Sst-Pten-WT), $\mathrm{Sst}^{-C r e}{ }^{+/+} /$Pten $^{\mathrm{fl} /+}$ (Sst-Pten-Het), and Sst-Cre ${ }^{+/+} /$Pten $^{\mathrm{fl} /}$ ${ }^{\mathrm{fl}}$ (Sst-Pten-KO). We also verified that Pten was specifically reduced and deleted in Sst-positive neurons of SstPten-Het and Sst-Pten-KO mice, respectively (Additional file 1: Figure S2), and there was no pre-existing side preference in the Sst-Pten mice (Additional file 1: Figure S3B). We found that all three genotypes of Sst-Pten mice spent more time in the interaction areas for social partners in the sociability test (Fig. 1d). This result was also confirmed by the preference index for sociability which was slightly reduced in Sst-Pten-Het mice while increased

\footnotetext{
(See figure on next page.)

Fig. 1 PV-Pten-KO and Sst-Pten-KO mice displayed social deficits in the modified three-chamber social test. a Schematic diagrams of the modified three-chamber social test. $\mathbf{b}$ All three genotypes of PV-Pten mice showed significant social preference between objects and mice (social partners) (WT: $n=26, W=325, p<0.0001$, Wilcoxon test; Het: $n=17, t_{(16)}=5.065, p=0.0001$, paired $t$-test; KO: $n=21, W=151, p=0.0071, W i l c o x o n$ test). There is no significant difference in preference index for social stimulus among these three genotypes of PV-Pten mice $\left(F_{(2,61)}=0.213, p=0.809\right.$, one-way ANOVA). c Only PV-Pten-WT mice displayed significant preference between familiar and novel mice in the social novelty test (WT: $n=26$, $t_{(25)}=6.142, p<0.0001$; Het: $n=17, t_{(16)}=0.7481, p=0.4652 ; \mathrm{KO}: \mathrm{n}=21, t_{(20)}=0.9664, p=0.3454$; Paired $t$-test). The preference index for social novelty significantly reduced in PV-Pten-Het and PV-Pten-KO mice compared with PV-Pten-WT mice $\left(F_{(2,61)}=7.402, p=0.0011\right.$, one-way ANOVA; Het vs. WT: $p=0.0427$, KO vs. WT: $p=0.0011$, Tukey's post hoc test). $\mathbf{d}$ All three genotypes of Sst-Pten mice showed significant social preference between objects and social partners (WT: $\mathrm{n}=22, t_{(21)}=4.570, p=0.0002$, paired $t$-test; Het: $\mathrm{n}=29, \mathrm{~W}=221.0, p=0.0157, \mathrm{Wilcoxon}$ test; KO: $\mathrm{n}=24$, $W=254.0, p<0.0001$; Wilcoxon test). The preference index for social stimulus reduced slightly in Sst-Pten-Het mice and increased in Sst-Pten-KO mice, but none of these changes reached statistical significance in post hoc comparisons ( $p=0.0008$, Kruskal-Wallis test; Het vs. WT: $p=0.2287$, KO vs. WT: $p=0.0855$, Dunn's post hoc test). e Sst-Pten-Het and Sst-Pten-KO mice displayed deficits in the social novelty test $\left(\right.$ WT: $\mathrm{n}=22, t_{(21)}=5.269$, $p<0.0001$, paired $t$-test; Het: $\mathrm{n}=29, \mathrm{~W}=99, p=0.2941$, Wilcoxon test; $\mathrm{KO}: \mathrm{n}=24, t_{(23)}=1.775, p=0.0891$, paired $t$-test). The preference index for social novelty significantly reduced in Sst-Pten-Het and Sst-Pten-KO mice ( $p=0.0311$, Kruskal-Wallis test; Het vs. WT: $p=0.0442, \mathrm{KO}$ vs. WT: $p=0.0384$, Dunn's post hoc test). The preference index was calculated as described in the methods. Circles represent data from individual animals, and bar graphs indicate mean + SEM. *: significant; n.s.: not significant
} 


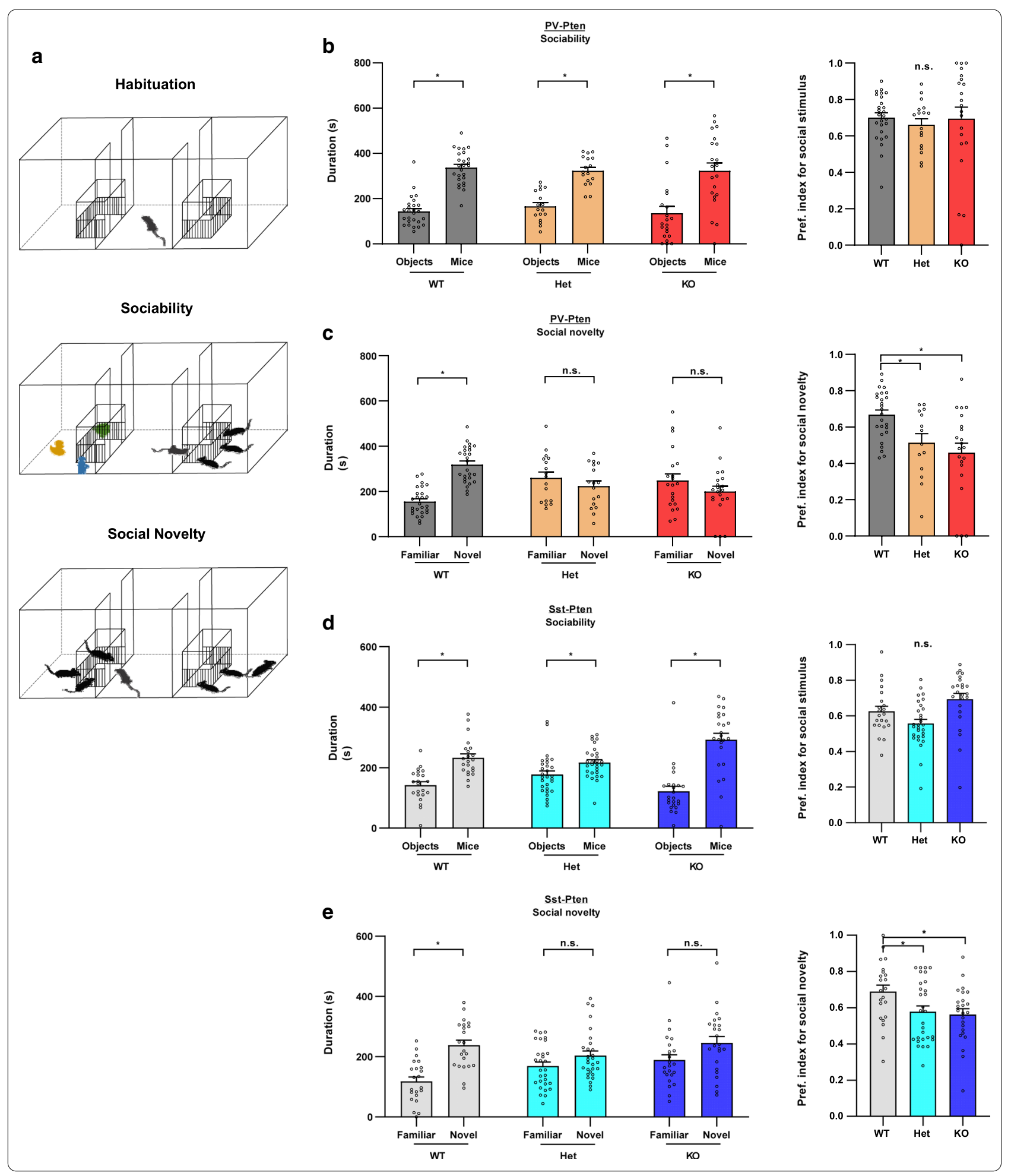

in Sst-Pten-KO mice, but these changes were not significant in post hoc comparisons (Fig. 1d). In the social novelty test, only Sst-Pten-WT but not -Het or -KO mice spent more time in the interaction zones for novel social partners (Fig. 1e). Consistently, the preference indexes for social novelty was reduced in both Sst-Pten-Het and -KO mice (Fig. 1e). Thus, Sst-Pten-Het and -KO mice displayed normal sociability but exhibited impairment in 
social novelty behavior. Collectively, elimination of Pten in either PV-neurons or Sst-neurons resulted in reduced social behaviors, particularly social novelty preference.

\section{Deficits of locomotion and motor coordination and increased repetitive behaviors in PV-Pten and Sst-Pten mice}

We then examined the contribution of Pten mutations in PV- and Sst-neurons to deficits in locomotor behaviors. 30-min open field tests were performed in PV-Pten mice (Fig. 2a). PV-Pten-Het but not -KO mice traveled significantly longer distances compared to PV-Pten-WT mice (Fig. 2b). When examined in 5-min intervals, the distance moved in PV-Pten-WT and -Het mice declined slightly over the 30-min testing period, and PV-Pten-Het mice consistently traveled longer distances in each interval. PV-Pten-KO mice maintained the same rate of activity over the 30 -min testing time $(\mathrm{p}=0.903$, Pearson correlation test), but no statistical difference compared to PVPten-WT mice (Fig. 2c). We examined the locomotor activity in home cages as well. Consistent to the result in the open-field tests, PV-Pten-Het mice but not PV-PtenKO mice exhibited increased locomotor activity (Fig. 2d and e). Thus, PV-Pten-Het mice exhibited elevated locomotor activity both in the novel open field and home cages. In Sst-Pten mice, the locomotor activity was not different among genotypes (Fig. 2f), and was confirmed by measurements across 5 -min intervals (Fig. 2g). SstPten mice also performed normally in the home-cage activity test (Fig. $2 \mathrm{~h}$ and i).

We next examined self-grooming during the open field test for repetitive behaviors. PV-Pten-KO mice exhibited an increase of grooming bouts (Fig. 3a) and spent more time in self-grooming in the open field (Fig. 3b). Similarly, the number of grooming bouts and total time of self-grooming were increased in Sst-Pten-KO mice (Fig. 3c and d). These data suggest that deletion of Pten in
PV-neurons or Sst-neurons induces repetitive behaviors, a core-domain of autism behaviors.

We further evaluated the motor coordination/learning in PV-Pten mice using the rotarod test (Fig. 4a). Mice were trained on an accelerating rotarod for 6 trials over two days ( 3 trials per day). The latency to fall was significantly reduced in PV-Pten-Het and PV-Pten-KO mice over the 6-trial training (Fig. 4b). Particularly, motor coordination was severely impaired in PV-Pten-KO mice. They were barely able to stay on the stationary rotarod (Fig. 4b). We next analyzed the learning index (ratio of latency to fall between the 6th trial and the 1st trial) and found that knockout of Pten in PV-neurons impaired motor learning in the rotarod test (Fig. 4c). Similarly, knockout of Pten in Sst-neurons impaired rotarod performance and motor learning as well (Fig. 4d and e). Collectively, knockdown or knockout of Pten in PV-neurons or Sst-neurons impairs motor coordination and learning.

We then conducted marble burying tests in PV-Pten and Sst-Pten mice (Fig. 4f). PV-Pten-KO mice buried significantly fewer marbles than PV-Pten-WT mice did (Fig. 4g), but there was no difference in the marble burying task among the three genotypes of Sst-Pten mice (Fig. 4h). Since the burying of marbles entails physical motor activity, reduced marble burying activity in PVPten-KO mice may be mainly resulted from impaired motor coordination in this type of mice (Fig. 4b).

\section{Anxiolytic-like behavior in PV-Pten-KO mice and anxiety-like behavior in Sst-Pten-KO mice}

Finally, we examined anxiety-like behaviors in mice with Pten deletion in inhibitory neurons using elevated plus maze tests (Fig. 5a) and open field tests (Fig. 5b). In the elevated plus maze, PV-Pten-KO mice spent increased percentage of time in the open arms and decreased percentage of time in the closed arms, compared to PV-Pten-WT and PV-Pten-Het mice (Fig. 5c and d). Consistently, in the open field test,

\footnotetext{
(See figure on next page.)

Fig. 2 PV-Pten-Het mice exhibited hyper-locomotion in the open field test. a The schematic diagram of the open field test. b PV-Pten-Het mice traveled a longer distance than PV-Pten-WT mice during the 30-min test (WT: $n=25$, Het: $n=17, K O ; n=26 ; F_{(2,65)}=7.244$, $p=0.001$, one-way ANOVA; Het vs. WT: $p<0.001$, KO vs. WT: $p=0.236$, Tukey's post hoc test). $\mathbf{c}$ When the total distance of PV-Pten mice was analyzed in 5-min time bin, PV-Pten-Het mice displayed hyperlocomotion ( $p=0.0012$, genotype effect, two-way rmANOVA; Het vs. WT: $p<0.001$, KO vs. WT: $p=0.236$, Tukey's post hoc test). d PV-Pten-Het mice exhibited increased travel distance in 30-min home-cage activity (WT: $n=6, H e t: n=8, K O ; n=5 ; F_{(2,16)}=12.93$, $p=0.0005$, one-way ANOVA; Het vs. WT: $p=0.036 ; \mathrm{KO}$ vs. WT, $p=0.087$, Tukey's post hoc test). e Home-cage activity was analyzed in a 5-min time bin. PV-Pten-Het mice displayed hyperlocomotion in home cages ( $p=0.0001$, genotype effect, two-way rmANOVA; Het vs. WT: $p=0.0017$, KO vs. WT: $p=0.4685$, Tukey's post hoc test). $\mathbf{f}, \mathbf{g}$ There was no significant difference in total distance moved (d; WT: $n=12$, Het: $n=16, K O$ : $\mathrm{n}=19 ; F_{(244)}=0.313, p=0.733$, one-way ANOVA) or distance moved in 5-min time bin (e) in Sst-Pten mice ( $p=0.7002$, genotype effect, two-way rmANOVA). $\mathbf{h}, \mathbf{i}$ In home-cage activity test, there was no difference in the total distance traveled (h; WT: $n=4$, Het: $n=17, K O: n=8 ; F_{(2,26)}=0.088$, $p=0.9164$, one-way ANOVA) or the distance moved in 5 -min time bin (i; $p=0.9164$, genotype effect, two-way rmANOVA) among the three genotypes of Sst-Pten mice. In $\mathbf{b}, \mathbf{d}$, $\mathbf{f}$ and $\mathbf{h}$, circles represent data from individual animals, and bar graphs indicate mean $+S E M$. In $\mathbf{c}, \mathbf{e}, \mathbf{g}$ and $\mathbf{i}$, data are represented as mean \pm SEM. *: significant; $n$.s.: not significant
} 


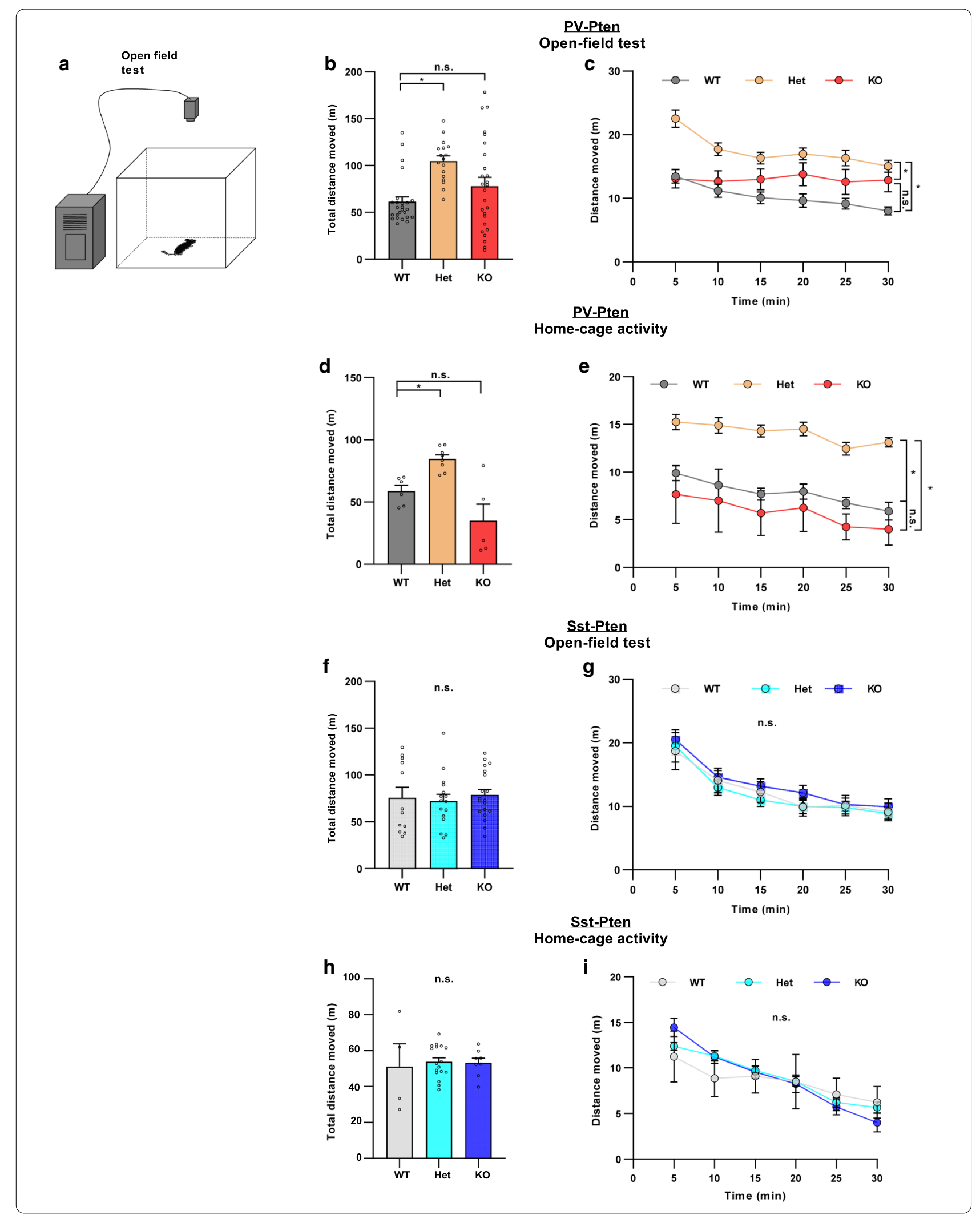




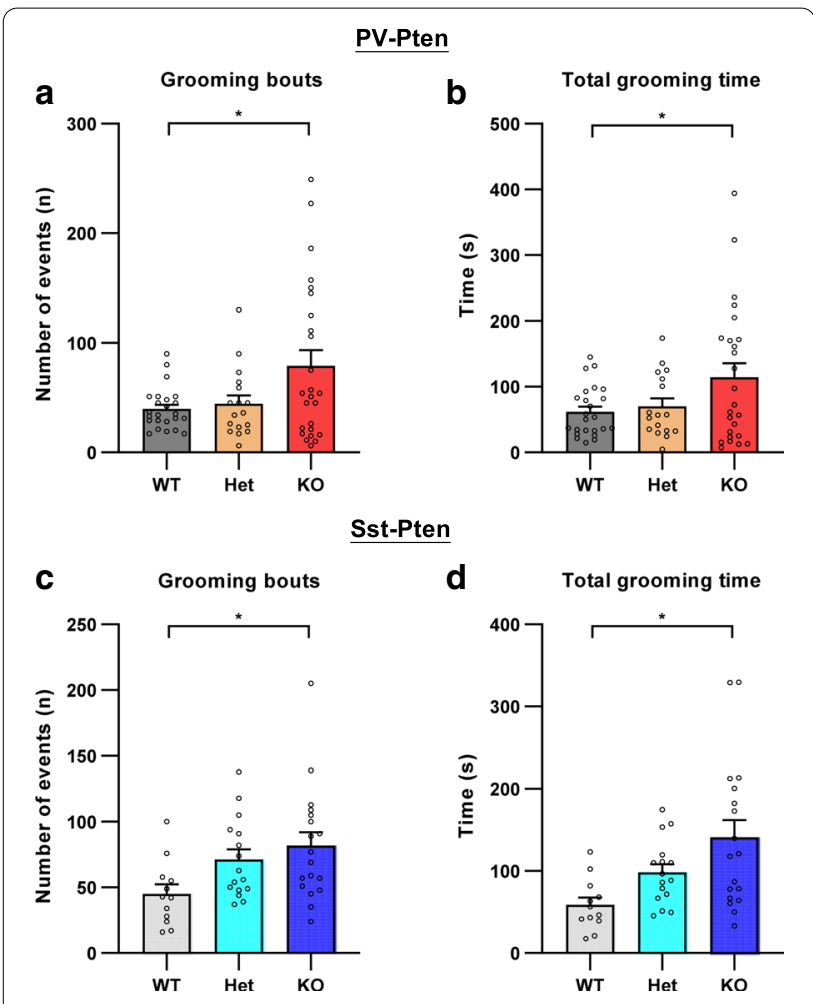

Fig. 3 Increased self-grooming in PV-Pten-KO and Sst-Pten-KO mice. a The number of self-grooming bouts increased in PV-Pten-KO mice (WT: $\mathrm{n}=24$, Het: $\mathrm{n}=17, \mathrm{KO}: \mathrm{n}=25 ; F_{(2,63)}=4.758, p=0.012$, one-way ANOVA; KO vs. WT: $p=0.015$, Tukey's post hoc test). $\mathbf{b}$ The total time of self-grooming increased in PV-Pten-KO mice (WT: $n=24$, Het: $\mathrm{n}=17, \mathrm{KO}: \mathrm{n}=25 ; F_{(2,63)}=3.635, p=0.032$, one-way ANOVA; KO vs. WT: $p=0.036$, Tukey's post hoc test). $\mathbf{c}$ The number of self-grooming bouts increased in Sst-Pten-KO mice (WT: $n=12$, Het: $n=16$, $\mathrm{KO}: \mathrm{n}=18 ; F_{(2,43)}=4.025, p=0.0250$, one-way ANOVA; KO vs. WT: $p=0.0199$, Tukey's post hoc test). $\mathbf{d}$ The total time of self-grooming increased in Sst-Pten-KO mice (WT: $n=12$, Het: $n=16, K O: n=18$;

$F_{(2,43)}=6.249, p=0.004$, one-way ANOVA; KO vs. WT: $p=0.003$, Tukey's post hoc test). Circles represent data from individual animals, and bar graphs indicate mean + SEM. *: statistically significant

PV-Pten-KO mice spent increased time in the center area (Fig. 5e), and decreased time in the corner area of the open field compared to PV-Pten-WT mice (Fig. 5f). In contrast, Sst-Pten-KO mice showed a reduced percentage of time in the open arms and an increased percentage of time in the closed arms (Fig. $5 \mathrm{~g}$ and h). We did not observe any difference of time spent in the center or corner areas among the three genotypes of Sst-Pten mice (Fig. 5i and j). Thus, we concluded that Pten knockout in PV-neurons induces anxiolytic-like behavior while Pten knockout in Sst-neurons evokes anxiety-like behavior.

\section{Discussion}

Our study revealed that conditional deletion of Pten in either PV-neurons or Sst-neurons results in autismlike core symptoms, including reduced social novelty preference and presence of repetitive behaviors (selfgrooming). In addition, conditional Pten knockout in PV-neurons causes differences in behaviors that are characteristic symptoms often observed in autism, such as hyperactivity and impairment in motor coordination/ learning. Similarly, conditional Pten knockout in Sst-neurons gives rise to disrupted motor coordination/learning. Interestingly, PV-Pten-KO mice displayed anxiolytic-like behaviors, whereas Sst-Pten-KO mice showed anxietylike behaviors. These findings provide the behavioral evidence that Pten mutation in PV-neurons or Sst-neurons sufficiently induces ASD-related behaviors.

The mice used in this study all had homozygous Cre and different copies of Pten in PV-neurons or Sst-neurons. Reduced somatostatin expression in homozygous Sst-Cre mice has been previously reported [31], which might potentially impact behavioral performance. We compared behaviors in PV-Pten-WT mice and Sst-PtenWT mice with those in $\operatorname{Pten}^{\mathrm{f} / \mathrm{fl}}$ mice, and found that PV-Pten-WT and Sst-Pten-WT mice did not exhibit any difference in most behavioral tests, except that Sst-PtenWT mice displayed increased time spent in the center of the open field test (Additional file 1: Figure S4). Since Cre expression is just the background for all genotypes of mice used here, our conclusion in this study should not be affected by the change of baseline behavioral performance in Sst-Pten-WT mice. We were also wondering whether the different ages of mice performed similarly in the behavioral tests. By performing correlation tests, we found that there was no relationship between the age and behavioral performance in all behavioral tests (Additional file 1: Figures S5 and S6). Additionally, the prevalence of autism is different in sex [32] and our previous study in animal models also showed the sex difference in behaviors of Slit3-KO mice [33]. However, we did not found any sex effect in the behavioral phenotypes of PV-Pten or Sst-Pten mice (Additional file 1: Figure S7 and S8).

Mice with deletion of several other genes (Shank2, mGluR5 and Lom4) in PV-neurons exhibit autism-related behaviors of varying severity [34-36], supporting that the function of PV-neurons is an important determinant of autism behaviors. Autism-relevant behavioral phenotypes are also reported in mice with conditional knockout of MeCP2 (Rett syndrome) or Scn1a (Dravet syndrome) in PV-neurons but not Sst-neurons [37, 38]. In our results, conditional knockout of Pten in either PVor Sst-neurons resulted in autism-like core behaviors, further suggesting that Pten plays an important role in the function of these two subtypes of neurons. 


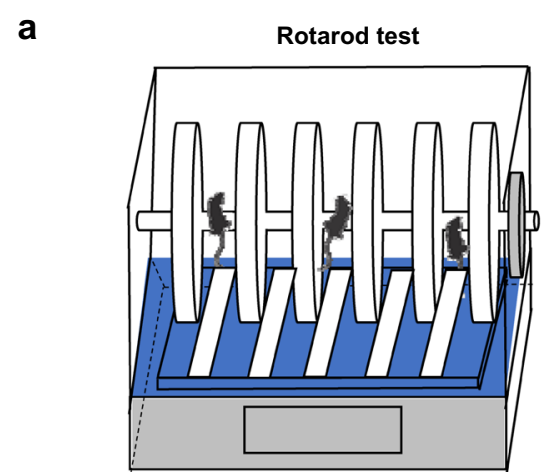

b

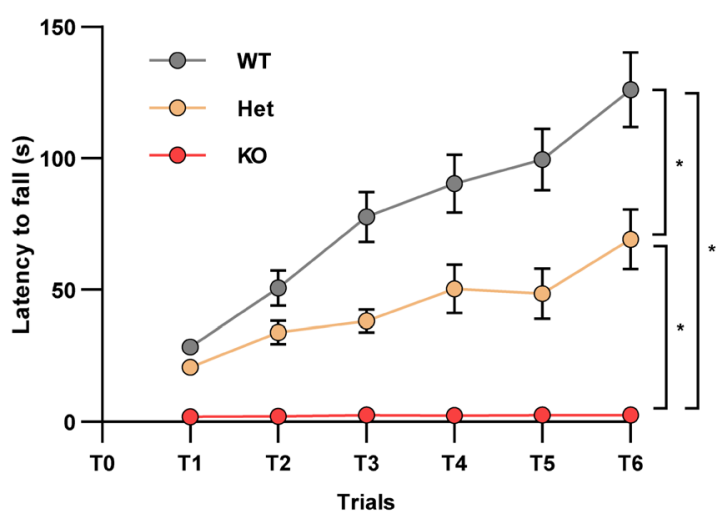

d

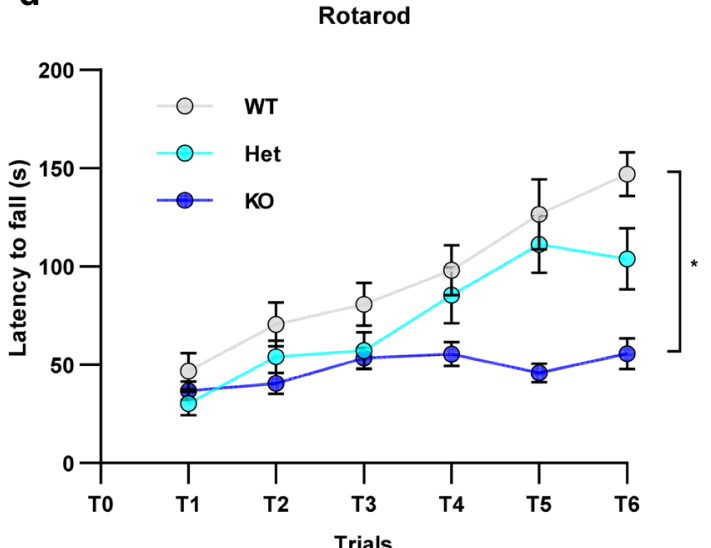

PV-Pten

C

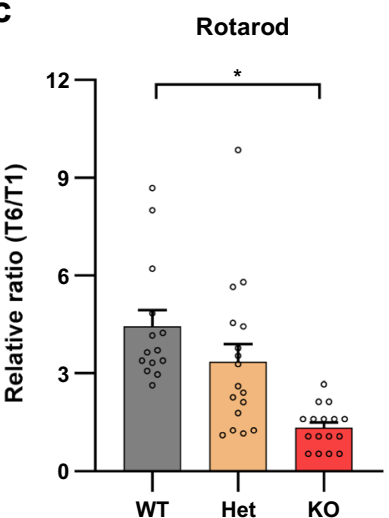

g

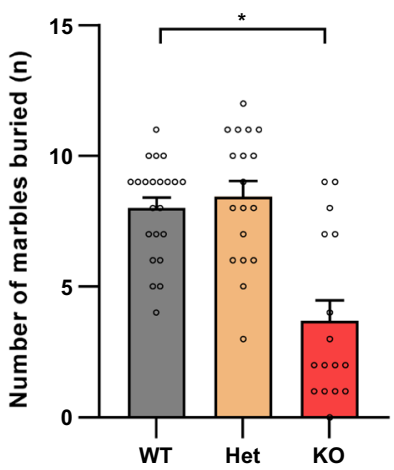

Sst-Pten

e

Rotarod

h

Marble burying task

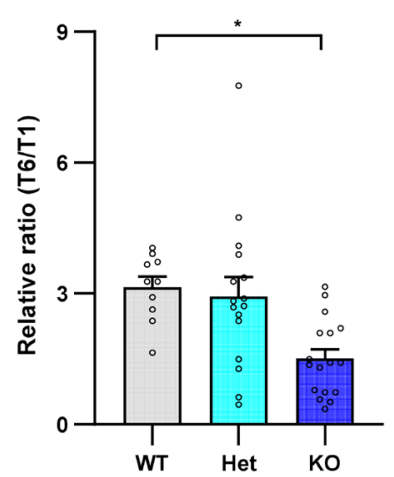

f

Marble burying task

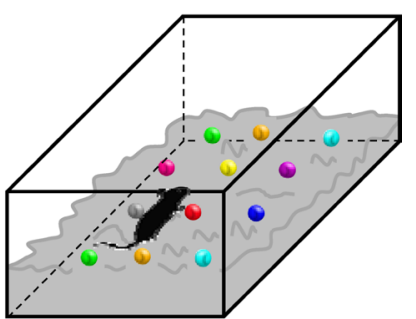

Fig. 4 Impaired motor function in PV-Pten-KO and Sst-Pten-KO mice. a The schematic diagram of the rotarod test. b PV-Pten-Het and PV-Pten-KO mice showed a shorter latency to fall off the rotarod than PV-Pten-WT mice over the 6-trials training (WT: $n=14$, Het: $n=17, K O: n=14 ; p<0.0001$, genotype effect, two-way rmANOVA; Het vs. WT: $p<0.0001$, KO vs. WT: $p<0.0001$, Het vs. KO: $p<0.0001$, Tukey's post hoc test). c The learning index (T6/T1) was significantly reduced in PV-Pten-KO mice $\left(F_{(2,44)}=12.435, p<0.001\right.$, one-way ANOVA; KO vs. WT: $p<0.001$, Tukey's post hoc test). d Sst-Pten-KO mice showed a shorter latency to fall off the rotarod than Sst-Pten-WT mice over the 6-trials training (WT: $n=10, H e t: n=16, K O$ : $\mathrm{n}=17 ; p=0.0005$, genotype effect, two-way rmANOVA; KO vs. WT: $p=0.0004$, Tukey's post hoc test). e The learning index (T6/T1) was significantly reduced in Sst-Pten-KO mice ( $F_{(2,40)}=7.372, p=0.0019$, one-way ANOVA; KO vs. WT: $p=0.0065$, Tukey's post hoc test). $\mathbf{f}$ The schematic diagram of the marble burying test. $\mathbf{g}$ PV-Pten-KO mice buried significantly fewer marbles compared to PV-Pten-WT mice (WT: $\mathrm{n}=22, \mathrm{Het}: \mathrm{n}=18, \mathrm{KO}$ : $\mathrm{n}=16 ; p<0.001$, Kruskal-Wallis test; KO vs. WT, $p=0.001$, Dunn's post hoc test). $\mathbf{h}$ There was no difference in the number of marbles buried among the three genotypes of Sst-Pten mice (WT: $\mathrm{n}=7$, Het: $\mathrm{n}=8, \mathrm{KO}: \mathrm{n}=10 ; p=0.406$, Kruskal-Wallis test). In $\mathbf{c}, \mathbf{e}, \mathbf{g}$ and $\mathbf{h}$, circles represent data from individual animals, and bar graphs indicate mean + SEM. In $\mathbf{b}$ and $\mathbf{d}$, data are represented as mean \pm SEM. *: significant; $n$.s.: not significant 

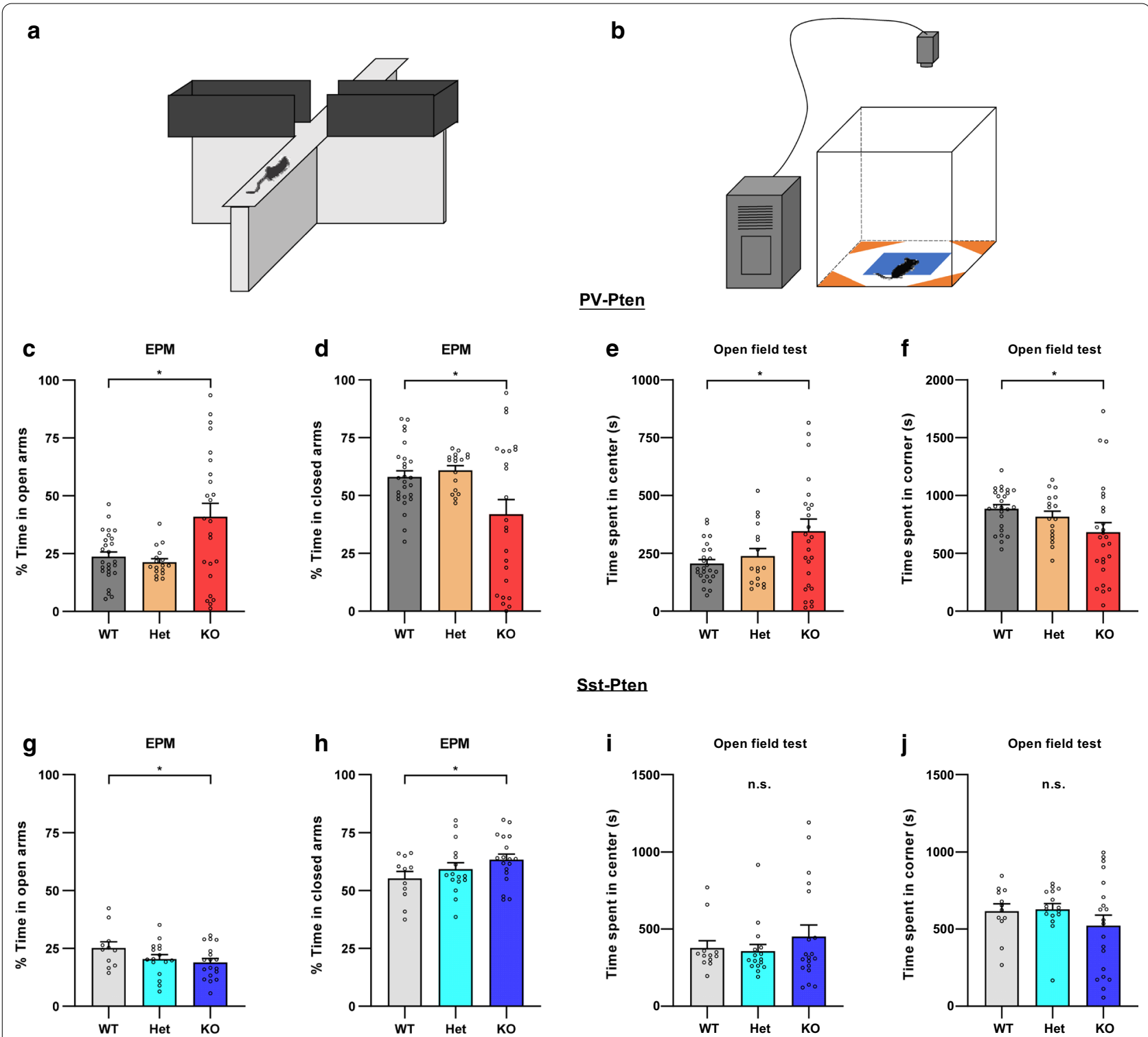

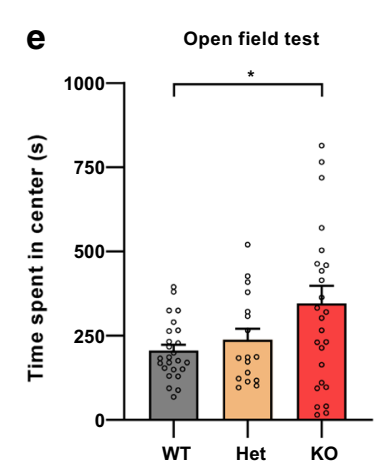

Sst-Pten

b

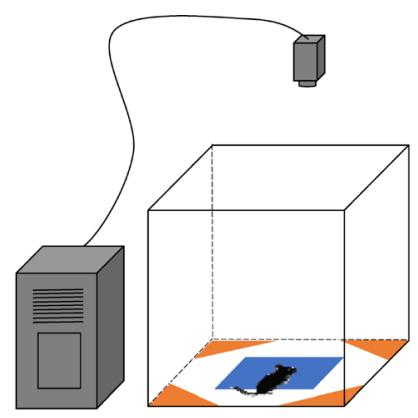

$\underline{\text { PV-Pten }}$

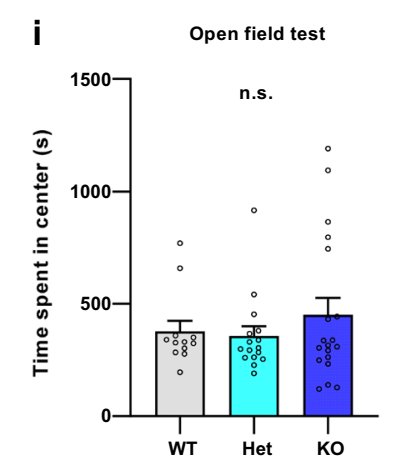

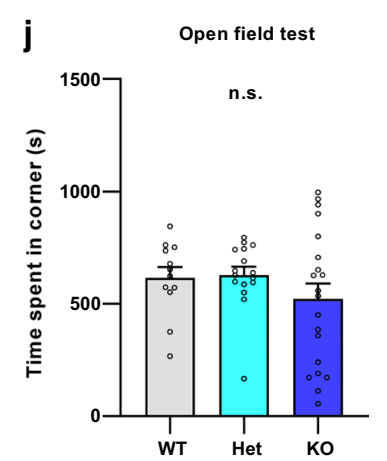

Fig. 5 PV-Pten-KO mice showed anxiolytic behaviors while Sst-Pten-KO mice displayed anxiety-like behaviors, compared to their WT mice. a The schematic diagram of the elevated plus-maze test (EPM). $\mathbf{b}$ The schematic diagram of the center (blue) and corner area (orange) in the open field test. c PV-Pten-KO mice spent more percentage of time in the open arms compared to PV-Pten-WT and PV-Pten-Het mice (WT: $n=26$, Het: $n=17$, KO: $\mathrm{n}=24 ; F_{(2,64)}=7.373, p=0.001$, one-way ANOVA; KO vs. WT: $p=0.005$, Tukey's post hoc test). $\mathbf{d}$ PV-Pten-KO mice spent less percentage of time in the closed arms compared to PV-Pten-WT and PV-Pten-Het mice $\left(F_{(2,64)}=5.310, p=0.007\right.$, one-way ANOVA; KO vs. WT: $p=0.023$, Tukey's post hoc test). $\mathbf{e}, \mathbf{f} I \mathrm{n}$ the open field test, PV-Pten-KO mice spent more time in the center area (e) and less time in the corner area (f), compared to PV-Pten-WT mice (WT: $\mathrm{n}=25$, Het: $\mathrm{n}=17, \mathrm{KO}: \mathrm{n}=26$; Center: $p=0.024$, one-way ANOVA; KO vs. WT: $p=0.023$, Tukey's post hoc test; Corner: $p=0.016$, KruskalWallis test; KO vs. WT: $p=0.009$, Dunn's post hoc test). $\mathbf{g}$ Sst-Pten-KO mice spent less percentage of time in the open arms compared to Sst-Pten-WT mice (WT: $\mathrm{n}=11$, Het: $\mathrm{n}=16, \mathrm{KO}: \mathrm{n}=17 ; F_{(2,34)}=3.984, p=0.028$, one-way ANOVA; KO vs. WT: $p=0.026$, Tukey's post hoc test). $\mathbf{h}$ Sst-Pten-KO mice spent more percentage of time in the closed arms compared to Sst-Pten-WT mice $\left(F_{(2,34)}=3.596, p=0.038\right.$, one-way ANOVA; KO vs. WT: $p=0.031$, Tukey's post hoc test). $\mathbf{i}, \mathbf{j} \mid \mathrm{n}$ the open field test, there was no difference in time spent in the center (i) or corner area (j) among the three genotypes of Sst-Pten mice (WT: $\mathrm{n}=12$, Het: $\mathrm{n}=16, \mathrm{KO}: \mathrm{n}=19$; Center: $p=0.851$, Kruskal-Wallis test; Corner: $p=0.502$, Kruskal-Wallis test). Circles represent data from individual animals, and bar graphs indicate mean + SEM. *: significant; n.s.: not significant

Three-chamber social tests are commonly used to examine sociability and social novelty preference in mice [39]. The sociability test is to assess the tendency of initiating social contact and remaining proximal to an unfamiliar conspecific, whereas the social novelty test is to evaluate the tendency of approaching novel 
mice, the discrimination between familiar and novel mice, and the recognition/memorization of the mouse that has interacted before [40]. PV-Pten and Sst-Pten mice exhibited deficits mainly in social novelty preference. Multiple brain regions could be involved in the social deficits observed in these mice [41]. The prefrontal cortex (PFC) is one of such brain structures that are well established for social behaviors [42]. PV-neurons and Sst-neurons in medial prefrontal cortex (mPFC) specifically respond to social stimuli $[43,44]$. Social-evoked responses in PV-neurons are diminished in Cntnap2-KO mice which display deficits in sociability [43]. Consistently, optogenetic activation of PV-neurons in mPFC rescues deficits of sociability and social novelty preference in autism mouse models [43, 45]. However, evidence supporting a relationship between PV-neurons in $\mathrm{MPFC}$ and social deficits is not unequivocal [46].

The hippocampus is another brain region which is involved in social interaction, particular social memory, a process that is critical for social novelty preference [47, 48]. PV-neurons in the ventral hippocampus (vHipp) exhibited increased activity to novel social partners compared to familiar ones, and inactivation of PV-neurons but not Sst-neurons in vHipp disrupts social novelty preference [49]. Conversely, another study reported that knockdown Sst but not PV in vHipp disrupted social interaction [50]. Our data, however, showed that both PV- and Sst-neurons are important for social novelty preference, indicating that multiple brain regions might contribute to this type of social behaviors.

The cerebellum was recently implicated in social behaviors [51, 52]. PV and Sst are expressed in Purkinje cells (PC) of the cerebellum [53-55]. Abnormal PC function has been linked to social deficits in mice with conditional deletion of autism-risk genes in PCs [56-59]. However, conditional Pten knockout in Purkinje cells results in autism-related behaviors, such as less self-grooming and reduced sociability [28], different phenotypes to those exhibited in PV-Pten-KO mice (increased self-grooming and reduced social novelty preference). This discrepancy may be explained by the fact that Cre-recombinase activity in PV-Cre mice is not only present in Purkinje cells but also in cerebellar interneurons [53,60], indicating that cerebellar interneurons may contribute to behavioral phenotypes in PV-Pten-KO mice as well. In addition, other brain regions, such as the amygdala [61, 62], ventral tegmental area (VTA)-to nucleus accumbens (NAc) projection [63], and the striatum [64, 65] all have been reported to be involved in social behaviors.

Mutation of Pten in PV-neurons may contribute to hyperactivity, a common comorbidity of autism [66]. However, only PV-Pten-Het mice exhibited hyperactivity in the open field test. Both PV-Pten-KO mice and
Sst-Pten-KO mice displayed impaired motor coordination/learning in accelerating rotarod tasks and increased repetitive behaviors (self-grooming) in open field tests. Especially, PV-Pten-KO mice were barely able to stay on the rotarod. This severe deficit of motor coordination in PV-Pten-KO mice may mask the hyperactivity resulting from the Pten knockout in PV-neurons. The cerebellum and striatum, two brain structures critical for motor coordination/learning and repetitive behaviors [67-69], may account for these behavioral phenotypes in PVPten and Sst-Pten mice. Additionally, Pten knockout in Purkinje cells results in impaired motor coordination/ learning [28], and inhibition of PCs in cerebellar right crus I results in repetitive behaviors [59]. Moreover, Pten regulates striatal dopamine signaling [70, 71]. All these lines of evidence indicate that motor deficits and repetitive behaviors in PV-Pten and Sst-Pten mice may result from the functional change of PV-and Sst-neurons in the cerebellum and/or striatum; however, further investigations are needed.

Our results showed that PV-Pten-KO mice exhibited anxiolytic-like behaviors while Sst-Pten-KO mice displayed anxiety-like behaviors. Activation of PV-positive neurons in the dentate gyrus results in anxiolytic behaviors [72]. Interestingly, PV activity in the nucleus accumbens shell (sNAc) negatively correlates with the time of open-arm exploration in EPM test [73]. The mechanisms underlying the differential effects of Pten knockout in these two subtypes of neurons on anxiety-like behaviors are not clear.

At the synaptic level, conditional knockout Pten from GABAergic cortical interneurons increases the synaptic input onto interneurons [74] and the inhibitory output onto glutamatergic neurons [22, 75]. Conversely, a single copy of Pten deletion from PV-neurons impairs the formation of perisomatic inhibition [76]. Though the underlying synaptic mechanisms in PV-Pten-KO and Sst-Pten-KO mice need further investigation, our results provide behavioral evidence that deletion of Pten in either subtype of inhibitory neurons elicits autismrelated phenotypes, suggesting a potential benefit from therapeutic strategies targeting either PV-neurons, Sstneurons, or both subtypes, depending on specific alterations in behavioral phenotype.

\section{Material and methods}

\section{Animals}

Pten $^{\mathrm{fl} /+}$, Sst-Cre ${ }^{+/-}$and PV-Cre ${ }^{+/-}$mice were purchased from Jaxson Laboratory (Stock \#: 006440, 013044 and 008069, respectively). All these strains were maintained on C57BL6/J background. We crossed Pten ${ }^{\mathrm{f} / \mathrm{fl}}$ mice with Sst-Cre ${ }^{+/+}$mice to generate $\mathrm{Sst}-\mathrm{Cre}^{+/-} / \mathrm{Pten}^{\mathrm{f} / /+}$ mice which were then backcrossed with Sst-Cre ${ }^{+/+}$mice to 
generate Sst-Cre ${ }^{+/+} /$Pten $^{\mathrm{fl} /+}$ breeders. By crossing the breeders we obtained three types of experimental mice, Sst-Cre ${ }^{+/+} /$Pten $^{+/+}$(Sst-Pten-WT), Sst-Cre ${ }^{+/+} /$Pten $^{\mathrm{f} / /+}$ (Sst-Pten-Het) and Sst-Cre ${ }^{+/+} /$Pten $^{\mathrm{f} / \mathrm{fl}}$ (Sst-Pten-KO) mice. They were all homozygous for Cre but expressed two, one or no copy of Pten in Sst neurons. Using the same breeding strategy, we generated PV-Cre ${ }^{+/+} / \mathrm{Pten}^{\mathrm{f} / /+}$ breeders and their offspring, $\mathrm{PV}-\mathrm{Cre}^{+/+} / \mathrm{Pten}^{+/+}(\mathrm{PV}-$ Pten-WT), PV -Cre ${ }^{+/+} /$Pten $^{\mathrm{fl} /+}$ (PV-Pten-Het) and PV-Cre ${ }^{+/+} /$Pten $^{\mathrm{f} / / \mathrm{fl}}$ (PV-Pten-KO) mice. Both male and female littermates at the age of 4-8 weeks were used for behavioral assessments. There was no significant correlation between the behavioral performance and ages in PVPten or Sst-Pten WT mice (Additional file 1: Figures S5 and S6). No significant sex effects were observed (Additional file 1: Figures S7 and S8). Age and sex-matched C57BL/6 mice (purchased from Veterinary Resources at the University of Maryland School of Medicine) were used as social partners in modified three-chamber social tests. All mice were housed 2-5 per cage in ventilated racks in a temperature- and humidity-controlled animal room on a $12 \mathrm{~h}$ light/dark cycle with lights on from 07:00 to $19: 00$ and cared by the AAALAC accredited program of the University of Maryland School of Medicine. Autoclaved rodent chow and water were available ad libitum. The experimental protocol was approved by the Institutional Animal Care and Use Committees at the Hussman Institute for Autism and the University of Maryland School of Medicine.

\section{Immunohistochemistry}

Animals were anesthetized with isoflurane and perfused transcardially with $0.9 \%$ saline solution. Brains were fixed in $4 \%$ paraformaldehyde. Double immunohistochemistry with either anti-parvalbumin made in guinea pig (1:3000 dilution; Swant; Catalog \# GP72) or anti-somatostatin made in rat (1:200 dilution; MilliporeSigma Cat.\# MAB354), and anti-PTEN made in rabbit (1:250 dilution; Cell Signaling; Catalog \# 9559T) were performed as described [77] in coronal sections of $40 \mu \mathrm{m}$ obtained with a vibratome (Leica VT 1000S). Appropriate Alexa fluor conjugated antibodies (Invitrogen) were used to detect primary antibodies. A negative control with normal goat serum in place of primary antibodies was performed on adjacent sections. Images were obtained with LSM 780 (Zeiss) confocal microscope. Imaging conditions were maintained over the different genotypes within each strain. Cells were counted in cortical layers III-IV $\left(\mathrm{PV}^{+}\right.$cells) or $\mathrm{V}-\mathrm{VI}\left(\mathrm{Sst}^{+}\right.$cells) from matching sections (Bregma $-1.7 \mathrm{~mm}$ ) of three mice per genotype.

PTEN expression levels were quantified by measuring the fluorescence intensity in parvalbumin or somatostatin positive cells with Fiji image analysis package [78].
After background subtraction, fluorescence intensities were normalized by the average PTEN fluorescence intensity in WT mice.

\section{Western blot}

Cerebellum (PV-Pten mice) or cortex (Sst-Pten mice) samples were homogenized in lysis buffer ( $\mathrm{PH} 7.4$; $10 \mathrm{mM}$ Tris $\mathrm{HCl}, 150 \mathrm{mM} \mathrm{NaCl}, 1 \mathrm{mM}$ EDTA, 1\% Triton X-100, $0.5 \%$ NP-40, $1 \mathrm{mM}$ sodium orthovanadate, $1 \mathrm{mM}$ PMSF) with protease inhibitor (Halt protease inhibitor, Pierce). $50 \mu \mathrm{g}$ of total protein was separated in $4-20 \%$ mini-Protean TGX Stain-Free precast gels (BioRad) and transferred to nitrocellulose membranes. Antibodies used included rabbit anti-PTEN, mouse anti $\beta$-Actin (Cell Signaling), and rabbit (Cell Signaling) and mouse (Novex) HRP-conjugated secondaries.

\section{Behavioral assessments}

Following procedures established in our previous work [33], a series of behavioral assessments were conducted. Due to poor reproductive performance of mutant mice, multiple behavioral assessments were performed on the same groups of mice. Each mouse was tested for only one behavioral assessment per day. The behavioral tests were performed in the order of potential stress elicited from the tasks during the tests: open field test, elevated plus maze test, modified three-chamber social test, marble burying task, and rotarod test. A large cohort of mice were used for all behavioral tests in the battery, except that a few mice died suddenly before the completion of the whole battery of tests. In addition, a second cohort of Sst-Pten mice were used for the social test only to balance the side preference, and a third cohort of mice were used for the home-cage activity test. Animals' activity during the behavioral assessments was recorded by a Logitech C920 HD Pro Webcam, with the Ethovision software (Noldus, RRID: SCR_002798) used for further offline analysis. All experiments were conducted during the light cycle with room lights at around $80 \mathrm{~lx}$ except for the elevated plus maze (around $8 \mathrm{~lx}$ ). All areas were cleaned with $70 \%$ ethanol between test sessions, including areas untouched by mice.

\section{Open field test}

Mice were placed in the center of an open field apparatus $(40 \times 40 \mathrm{~cm})$ and allowed to explore the field for $30 \mathrm{~min}$. The movement was tracked using the Ethovision XT software. Distance moved and time spent in the center area $(20 \times 20 \mathrm{~cm}$, square $)$ and corner area $(15 \times 15 \mathrm{~cm}$, triangle) were further analyzed. Self-grooming behaviors were also analyzed from the open field activity using mouse behavior recognition (Detection settings for each mouse: 
posture between 70 and $90 \%$, probability higher than 75\%) in the Ethovision XT.

\section{Home-cage activity test}

Home-cage activity was recorded and analyzed using Ethovision XT software. Mice were individually housed in a clean cage for at least $24 \mathrm{~h}$ for acclimation. Mouse behaviors were recorded in its home cage with the filter top removed for $30 \mathrm{~min}$. The distance moved was determined by off-line analyses of the video recordings.

\section{Elevated plus maze test}

The elevated plus maze was composed of two open arms $(30 \times 5 \mathrm{~cm})$ and two closed arms $(30 \times 5 \mathrm{~cm})$ with $15 \mathrm{~cm}$ wall height and $50 \mathrm{~cm}$ above the floor. Mice were individually placed in the center of the maze and facing one of the open arms. They were allowed to explore the open and closed arms of the maze for $10 \mathrm{~min}$. The duration of time in arms was recorded. The percentage of time in open arms (or closed arms) was calculated as the duration in open arms (or closed arms)/total exploring duration.

\section{Modified three-chamber social test}

We used a modified apparatus to examine the social behaviors [79]. The apparatus $[40(\mathrm{H}) \times 60(\mathrm{~L}) \times 20(\mathrm{~W})$ $\mathrm{cm}]$ was divided into three chambers $(20 \mathrm{~cm}$ long for each chamber) made from white Plexiglass. The side chambers and the center chamber were separated by white Plexiglass walls with a small gate $(10 \mathrm{~cm})$ in the middle. Square interaction areas $(10 \times 10 \mathrm{~cm})$ in each side chamber was formed by the gate and a three-sided fence made from clear Plexiglass (Fig. 1a). Test mice entered these two areas to interact with social partners. The two side chambers were covered by a transparent Plexiglass sheet during the three-session test. All three sessions were performed consecutively on one day. In the first session (habituation), the test mouse was placed in the center chamber and allowed to explore the chamber for $10 \mathrm{~min}$. At the end of the habituation session, the test mouse was gently guided back to the middle of the center chamber, and the small gates were blocked. During the second session, which was to test the sociability, three novel C57BL/6 mice and three objects were placed in one of the two side chambers, respectively. The side chamber for social partners or objects was randomly assigned by flipping a coin. The test mouse was allowed to explore the center chamber for $10 \mathrm{~min}$. After that, the test mouse was guided back to the middle of the center chamber again. In the third session, which was to test the social novelty preference, the objects were replaced by another set of three novel C57BL/6 mice, and the test mouse was allowed to explore the chamber for another
$10 \mathrm{~min}$. The duration that the animal spent in the interaction areas during each session was scored using the EthoVision XT software (Noldus). The preference index was calculated as the duration exploring the novel mice/ the total duration exploring the objects (or familiar mice) and the novel mice.

\section{Marble burying task}

Twelve glass marbles $(3 \times 4$ arrangement) were placed on the surface of the bedding $(5 \mathrm{~cm}$ deep $)$ in standard mouse cages. The cages were covered with filter top during the test. Each test mouse was placed in the cage and allowed to explore for $30 \mathrm{~min}$. The number of buried marbles was counted manually. The marble was checked from side of the cage. If the marble was buried more than $2 / 3$ deep, it was counted as buried.

\section{Rotarod test}

The two-session rotarod test was conducted on an accelerating rotarod (Panlab, Harvard Apparatus) over two days. Mice were allowed to become acclimated to the stationary rod for $60 \mathrm{~s}$ on the first day. Each test session consisted of three trials with 15-min inter-trial intervals (ITI). During each trial, mice were placed on the rotating rod $(4 \mathrm{rpm})$ facing away from the direction of rotation. The rotation speed was accelerated from 4 to $40 \mathrm{rpm}$ over $5 \mathrm{~min}$. If the mouse fell off the rod within $10 \mathrm{~s}$, the trial was repeated after $15 \mathrm{~min}$. The latency to fall was recorded as the time delay between the start of the trial and the moment when mice fell off the rod or made a complete revolution on the rod.

\section{Statistical analysis}

Data was analyzed with SPSS (v.20) and GraphPad Prism 9 Software. All datasets were tested for normality using the Shapiro-Wilk test. For datasets with normal distribution, two-tailed $t$-test was used for comparisons between two groups, and two-way ANOVA with repeated measures in one factor (rmANOVA) or one-way ANOVA was used for comparisons among three groups. Tukey's post hoc test was used for multiple comparisons after ANOVA test. For datasets with non-normal distribution, Wilcoxon matched-pairs signed rank test (Wilcoxon test) was used for comparisons between two groups (paired), and Kruskal-Wallis test with Dunn's post hoc test was used for comparisons among three groups. One sample $t$-test or Wilcoxon signed-rank test was used for comparing the mean (or median) of a dataset with a hypothetical number. $p$-value $<0.05$ was considered to be statistically significant. 


\section{Supplementary Information}

The online version contains supplementary material available at https://doi. org/10.1186/s13041-021-00731-8.

Additional file 1: Figure S1. PTEN expression in parvalbumin positive neurons of PV-Pten mice. Figure S2. PTEN expression in somatostatin positive neurons of Sst-Pten mice. Figure S3. No pre-existing side preference during the habituation session in modified three-chamber social tests for both PV-Pten and Sst-Pten mice. Figure S4. Cre expression in PV- or Sst-neurons did not change most behavioral performance. Figure S5. No significant correlation between behavioral performance and ages in PV-Pten-WT mice. Figure S6. No significant correlation between behavioral performance and ages in Sst-Pten-WT mice. Figure S7. No sex effect in open-field test and marble burying test was observed in PV-Pten or Sst-Pten mice. Figure S8. No sex effect in social test, rotarod test and EPM test was observed in PV-Pten or Sst-Pten mice.

\section{Abbreviations}

ASD: Autism spectrum disorder; PV: Parvalbumin; Sst: Somatostatin; PTEN: Phosphatase and tensin homolog on chromosome ten; PI3K/AKT/mTOR: Phosphatidylinositol 3-kinase/AKT/mammalian target of rapamycin; KO: Knockout; WT: Wildtype; PFC: Prefrontal cortex; mPFC: Medial prefrontal cortex; vHipp: Ventral hippocampus; PC: Purkinje cells.

\section{Acknowledgements}

We thank Dr. John Hussman, Dr. Gene Blatt, and Ms. Elizabeth Benevides for critical reading of the manuscript. We also thank Drs. Louis DeTolla and Turhan Coksaygan at the University of Maryland School of Medicine for providing veterinary and consulting services.

\section{Authors' contributions}

SS and AS collected the behavioral data. SS, AS and SH performed the data analyses. AS conducted the immunohistochemistry and Western blot. SS, AS and SH wrote the manuscript. SH conceived and designed the experiments with the help of SS and AS. All authors read and approved the final manuscript.

\section{Funding}

This work was supported by the Hussman Foundation Grant HIAS18001 to S.H.

\section{Availability of data and materials}

The datasets used and/or analyzed during the current study are available from the corresponding author on reasonable request.

\section{Ethics approval and consent to participate}

Not applicable.

\section{Consent for publication}

Not applicable.

\section{Competing interests}

The authors declare that they have no competing interests.

Received: 28 September 2020 Accepted: 5 January 2021 Published online: 27 January 2021

\section{References}

1. Marin O. Interneuron dysfunction in psychiatric disorders. Nat Rev Neurosci. 2012;13(2):107-20

2. Hussman JP. Suppressed GABAergic inhibition as a common factor in suspected etiologies of autism. J Autism Dev Disord. 2001:31(2):247-8.

3. Rapanelli M, Frick LR, Pittenger $C$. The role of interneurons in autism and Tourette syndrome. Trends Neurosci. 2017;40(7):397-407.

4. American Psychiatric Association. Diagnostic and statistical manual of mental disorders: DSM-5. 5th ed. Washington, DC: American Psychiatric Association; 2013.
5. Rudy B, Fishell G, Lee S, Hjerling-Leffler J. Three groups of interneurons account for nearly $100 \%$ of neocortical GABAergic neurons. Dev Neurobiol. 2011;71(1):45-61.

6. Zikopoulos B, Barbas H. Altered neural connectivity in excitatory and inhibitory cortical circuits in autism. Front Hum Neurosci. 2013;7:609.

7. Hashemi E, Ariza J, Rogers H, Noctor SC, Martinez-Cerdeno V. The number of parvalbumin-expressing interneurons is decreased in the prefrontal cortex in autism. Cereb Cortex. 2017;27(3):1931-43.

8. Ariza J, Rogers H, Hashemi E, Noctor SC, Martinez-Cerdeno V. The number of chandelier and basket cells are differentially decreased in prefrontal cortex in autism. Cereb Cortex. 2018;28(2):411-20.

9. Soghomonian JJ, Zhang K, Reprakash S, Blatt GJ. Decreased parvalbumin mRNA levels in cerebellar Purkinje cells in autism. Autism Res. 2017;10(11):1787-96.

10. Bridi MS, Park SM, Huang S. Developmental disruption of GABAARmeditated inhibition in Cntnap2 KO mice. eNeuro. 2017:4(5):1-14.

11. Sohal VS, Rubenstein JLR. Excitation-inhibition balance as a framework for investigating mechanisms in neuropsychiatric disorders. Mol Psychiatry. 2019;24(9):1248-57.

12. Lauber E, Filice F, Schwaller B. Parvalbumin neurons as a hub in autism spectrum disorders. J Neurosci Res. 2018;96(3):360-1.

13. Gregorian C, et al. Pten deletion in adult neural stem/progenitor cells enhances constitutive neurogenesis. J Neurosci. 2009;29(6):1874-86.

14. Stiles B, Groszer M, Wang S, Jiao J, Wu H. PTENless means more. Dev Biol. 2004;273(2):175-84.

15. Barker KT, Houlston RS. Overgrowth syndromes: is dysfunctional PI3-kinase signalling a unifying mechanism? Eur J Hum Genet. 2003;11(9):665-70.

16. Maehama T, Dixon JE. The tumor suppressor, PTEN/MMAC1, dephosphorylates the lipid second messenger, phosphatidylinositol 3,4,5-trisphosphate. J Biol Chem. 1998:273(22):13375-8.

17. Goffin A, Hoefsloot LH, Bosgoed E, Swillen A, Fryns JP. PTEN mutation in a family with Cowden syndrome and autism. Am J Med Genet. 2001;105(6):521-4.

18. Buxbaum JD, et al. Mutation screening of the PTEN gene in patients with autism spectrum disorders and macrocephaly. Am J Med Genet B. 2007;144B(4):484-91.

19. Hobert JA, Embacher R, Mester JL, Frazier TW 2nd, Eng C. Biochemical screening and PTEN mutation analysis in individuals with autism spectrum disorders and macrocephaly. Eur J Hum Genet. 2014;22(2):273-6.

20. McBride $\mathrm{KL}$, et al. Confirmation study of PTEN mutations among individuals with autism or developmental delays/mental retardation and macrocephaly. Autism Res. 2010;3(3):137-41.

21. Varga EA, Pastore M, Prior T, Herman GE, McBride KL. The prevalence of PTEN mutations in a clinical pediatric cohort with autism spectrum disorders, developmental delay, and macrocephaly. Genet Med. 2009;11(2):111-7.

22. Vogt D, Cho KKA, Lee AT, Sohal VS, Rubenstein JLR. The parvalbumin/ somatostatin ratio is increased in Pten mutant mice and by human PTEN ASD alleles. Cell Rep. 2015;11(6):944-56.

23. Ljungberg MC, Sunnen CN, Lugo JN, Anderson AE, D'Arcangelo G. Rapamycin suppresses seizures and neuronal hypertrophy in a mouse model of cortical dysplasia. Dis Model Mech. 2009;2(7-8):389-98.

24. Clipperton-Allen AE, Page DT. Decreased aggression and increased repetitive behavior in Pten haploinsufficient mice. Genes Brain Behav. 2015;14(2):145-57.

25. Kwon $\mathrm{CH}$, et al. Pten regulates neuronal arborization and social interaction in mice. Neuron. 2006;50(3):377-88.

26. Napoli E, et al. Mitochondrial dysfunction in Pten haplo-insufficient mice with social deficits and repetitive behavior: interplay between Pten and p53. PLoS ONE. 2012;7(8):e42504.

27. Lugo JN, et al. Deletion of PTEN produces autism-like behavioral deficits and alterations in synaptic proteins. Front Mol Neurosci. 2014;7:27.

28. Cupolillo D, et al. Autistic-like traits and cerebellar dysfunction in Purkinje cell PTEN knock-out mice. Neuropsychopharmacology. 2016:41(6):1457-66.

29. Clipperton-Allen AE, Page DT. Pten haploinsufficient mice show broad brain overgrowth but selective impairments in autism-relevant behavioral tests. Hum Mol Genet. 2014;23(13):3490-505. 
30. Page DT, Kuti OJ, Prestia C, Sur M. Haploinsufficiency for Pten and Serotonin transporter cooperatively influences brain size and social behavior. Proc Natl Acad Sci USA. 2009;106(6):1989-94.

31. Viollet C, et al. Somatostatin-IRES-Cre mice: between knockout and wildtype? Front Endocrinol (Lausanne). 2017;8:131.

32. Maenner MJ, et al. Prevalence of autism spectrum disorder among children aged 8 years - autism and developmental disabilities monitoring network, 11 sites, United States, 2016. MMWR Surveill Summ. 2020;69(4):1-12.

33. Park SM, Plachez C, Huang S. Sex-dependent motor deficit and increased anxiety-like states in mice lacking autism-associated gene Slit3. Front Behav Neurosci. 2018;12:261.

34. Lee $\mathrm{S}$, et al. Shank2 deletion in parvalbumin neurons leads to moderate hyperactivity, enhanced self-grooming and suppressed seizure susceptibility in mice. Front Mol Neurosci. 2018;11:209.

35. Barnes SA, et al. Disruption of mGluR5 in parvalbumin-positive interneurons induces core features of neurodevelopmental disorders. Mol Psychiatry. 2015;20(10):1161-72.

36. Zhang $\mathrm{L}$, et al. Hyperactivated PTP1B phosphatase in parvalbumin neurons alters anterior cingulate inhibitory circuits and induces autism-like behaviors. Nat Commun. 2020;11(1):1017.

37. Ito-Ishida A, Ure K, Chen H, Swann JW, Zoghbi HY. Loss of MeCP2 in parvalbumin-and somatostatin-expressing neurons in mice leads to distinct Rett syndrome-like phenotypes. Neuron. 2015;88(4):651-8.

38. Tatsukawa T, Ogiwara I, Mazaki E, Shimohata A, Yamakawa K. Impairments in social novelty recognition and spatial memory in mice with conditional deletion of Scn1a in parvalbumin-expressing cells. Neurobiol Dis. 2018;112:24-34

39. Yang M, Silverman $\mathrm{JL}$, Crawley JN. Automated three-chambered social approach task for mice. Curr Protoc Neurosci. 2011;Chapter 8: p. Unit 826.

40. Moy SS, et al. Sociability and preference for social novelty in five inbred strains: an approach to assess autistic-like behavior in mice. Genes Brain Behav. 2004;3(5):287-302.

41. Modi ME, Sahin M. A unified circuit for social behavior. Neurobiol Learn Mem. 2019;165:106920.

42. Scheggia D, et al. Somatostatin interneurons in the prefrontal cortex control affective state discrimination in mice. Nat Neurosci. 2020;23(1):47-60.

43. Selimbeyoglu A, et al. Modulation of prefrontal cortex excitation/inhibition balance rescues social behavior in CNTNAP2-deficient mice. Sci Transl Med. 2017;9(401):eaah6733.

44. Sun Q, et al. Ventral hippocampal-prefrontal interaction affects social behavior via parvalbumin positive neurons in the medial prefrontal cortex. iscience. 2020;23(3):100894.

45. Cao W, et al. Gamma oscillation dysfunction in mPFC leads to social deficits in neuroligin 3 R451C knockin mice. Neuron. 2018;97(6):1253-1260 e7.

46. Murray AJ, et al. Parvalbumin-positive interneurons of the prefrontal cortex support working memory and cognitive flexibility. Sci Rep. 2015;5:16778

47. Okuyama T, Kitamura T, Roy DS, Itohara S, Tonegawa S. Ventral CA1 neurons store social memory. Science. 2016;353(6307):1536-41.

48. Hitti FL, Siegelbaum SA. The hippocampal CA2 region is essential for social memory. Nature. 2014;508(7494):88-92.

49. Deng X, Gu L, Sui N, Guo J, Liang J. Parvalbumin interneuron in the ventral hippocampus functions as a discriminator in social memory. Proc Natl Acad Sci USA. 2019;116(33):16583-92.

50. Perez SM, Boley A, Lodge DJ. Region specific knockdown of parvalbumin or somatostatin produces neuronal and behavioral deficits consistent with those observed in schizophrenia. Transl Psychiatry. 2019;9(1):264.

51. Carta I, Chen CH, Schott AL, Dorizan S, Khodakhah K. Cerebellar modulation of the reward circuitry and social behavior. Science. 2019;363(6424):eaav0581.

52. Tsai PT. Autism and cerebellar dysfunction: evidence from animal models. Semin Fetal Neonatal Med. 2016;21(5):349-55.

53. Alcami P, Marty A. Estimating functional connectivity in an electrically coupled interneuron network. Proc Natl Acad Sci USA. 2013;110(49):E4798-807.

54. Asrican B, et al. Next-generation transgenic mice for optogenetic analysis of neural circuits. Front Neural Circ. 2013;7:160.

55. Taniguchi $\mathrm{H}$, et al. A resource of $\mathrm{Cre}$ driver lines for genetic targeting of GABAergic neurons in cerebral cortex. Neuron. 2011;71(6):995-1013.
56. Tsai PT, et al. Autistic-like behaviour and cerebellar dysfunction in Purkinje cell Tsc1 mutant mice. Nature. 2012;488(7413):647-51.

57. Reith RM, et al. Loss of Tsc2 in Purkinje cells is associated with autistic-like behavior in a mouse model of tuberous sclerosis complex. Neurobiol Dis. 2013;51:93-103.

58. Peter $\mathrm{S}$, et al. Dysfunctional cerebellar Purkinje cells contribute to autism-like behaviour in Shank2-deficient mice. Nat Commun. 2016;7:12627.

59. Stoodley CJ, et al. Altered cerebellar connectivity in autism and cerebellarmediated rescue of autism-related behaviors in mice. Nat Neurosci. 2017;20(12):1744-51.

60. Zhang B, Sudhof TC. Neuroligins are selectively essential for NMDAR signaling in cerebellar stellate interneurons. J Neurosci. 2016;36(35):9070-83.

61. Hong W, Kim DW, Anderson DJ. Antagonistic control of social versus repetitive self-grooming behaviors by separable amygdala neuronal subsets. Cell. 2014;158(6):1348-61.

62. Felix-Ortiz AC, Tye KM. Amygdala inputs to the ventral hippocampus bidirectionally modulate social behavior. J Neurosci. 2014;34(2):586-95.

63. Gunaydin LA, et al. Natural neural projection dynamics underlying social behavior. Cell. 2014;157(7):1535-51.

64. Lee Y, Kim H, Han PL. Striatal inhibition of MeCP2 or TSC1 produces sociability deficits and repetitive behaviors. Exp Neurobiol. 2018;27(6):539-49.

65. Rapanelli $\mathrm{M}$, et al. Targeted interneuron depletion in the dorsal striatum produces autism-like behavioral abnormalities in male but not female mice. Biol Psychiatry. 2017;82(3):194-203.

66. Simonoff E, et al. Psychiatric disorders in children with autism spectrum disorders: prevalence, comorbidity, and associated factors in a populationderived sample. J Am Acad Child Adolesc Psychiatry. 2008;47(8):921-9.

67. Whitehouse CM, Lewis MH. Repetitive behavior in neurodevelopmental disorders: clinical and translational findings. Behav Anal. 2015;38(2):163-78.

68. Kayakabe M, et al. Motor dysfunction in cerebellar Purkinje cell-specific vesicular GABA transporter knockout mice. Front Cell Neurosci. 2013;7:286.

69. Wang W, et al. Striatopallidal dysfunction underlies repetitive behavior in Shank3-deficient model of autism. J Clin Invest. 2017;127(5):1978-90.

70. Stavarache MA, Musatov S, McGill M, Vernov M, Kaplitt MG. The tumor suppressor PTEN regulates motor responses to striatal dopamine in normal and Parkinsonian animals. Neurobiol Dis. 2015;82:487-94.

71. Diaz-Ruiz $\mathrm{O}$, et al. Selective deletion of PTEN in dopamine neurons leads to trophic effects and adaptation of striatal medium spiny projecting neurons. PLOS ONE. 2009;4(9):e7027.

72. Zou D, et al. DREADD in parvalbumin interneurons of the dentate gyrus modulates anxiety, social interaction and memory extinction. Curr Mol Med. 2016;16(1):91-102.

73. Xiao Q, et al. A new GABAergic somatostatin projection from the BNST onto accumbal parvalbumin neurons controls anxiety. Mol Psychiatry. 2020. https ://doi.org/10.1038/s41380-020-0816-3.

74. Barrows CM, McCabe MP, Chen H, Swann JW, Weston MC. PTEN loss increases the connectivity of fast synaptic motifs and functional connectivity in a developing hippocampal network. J Neurosci. 2017;37(36):8595-611.

75. Southwell DG, et al. Interneuron transplantation rescues social behavior deficits without restoring wild-type physiology in a mouse model of autism with excessive synaptic inhibition. J Neurosci. 2020;40(11):2215-27.

76. Baohan A, Ikrar T, Tring E, Xu X, Trachtenberg JT. Pten and EphB4 regulate the establishment of perisomatic inhibition in mouse visual cortex. Nat Commun. 2016;7:12829.

77. Santi A, Genis L, Torres Aleman I. A coordinated action of blood-borne and brain insulin-like growth factor I in the response to traumatic brain injury. Cereb Cortex. 2018;28(6):2007-14.

78. Schindelin J, et al. Fiji: an open-source platform for biological-image analysis. Nat Methods. 2012;9(7):676-82.

79. Wu N, Wang Y, Pan Y-H, Yuan X. Identification of CDH11 as an ASD risk gene by matched-gene co-expression analysis and mouse behavioral studies. bioRxiv. 2020

\section{Publisher's Note}

Springer Nature remains neutral with regard to jurisdictional claims in published maps and institutional affiliations. 